\section{Aplicações do exercício intermitente de alta intensidade na síndrome metabólica}

\section{Applications of high-intensity intermittent exercise on metabolic syndrome}

\author{
Fabrício Boscolo Del Vecchio \\ Leony Morgana Galliano ${ }^{1}$ \\ Victor Silveira Coswig ${ }^{1}$
}

\section{Resumo}

Esta revisão de literatura apresenta os efeitos do exercício físico intermitente de alta intensidade (HIIT) em variáveis relacionadas à Síndrome Metabólica (SM). Após contextualização dos critérios de diagnóstico, explicitam-se resultados de intervenções para a SM, bem como as possibilidades de se obter benefícios com o HIIT. Foram recuperados 11 artigos que avaliaram efeitos agudos e 16 que mensuraram efeitos crônicos do exercício. Apontam-se melhoras na composição corporal (incluindo gordura visceral), em variáveis cardiovasculares (destaque para a potência aeróbia máxima) e em componentes metabólicos (principalmente relacionados à glicemia). Quando se compararam diferentes tipos de treinamento, intermitente e contínuo, observou-se indicações favoráveis ao HIIT para os diversos componentes da SM. Em conclusão, a partir deste estudo, observa-se que o HIIT pode ser relevante para a prevenção e tratamento da SM.

\section{Palavras-chave}

Exercício, Metabolismo, Obesidade, Hiperglicemia, Diabetes Mellitus, Hipertensão, Dislipidemia.

\begin{abstract}
This review provides information about the effects of high intensity intermittent training (HIIT) on variables related to the metabolic syndrome (MS). After the diagnoses criteria contextualization, results from exercise interventions on $M S$ are presented, as well as the possibility of achieving benefits with HIIT. Eleven studies that assessed the acute effects and 16 that measured the chronic effects of exercise were recovered. Studies showed improvements in body composition (including visceral fat), cardiovascular variables (especially aerobic power) and metabolic components (mainly related to glycemia). When comparing different types of training, intermittent and continuous, there are favorable indications for the HIIT in most of the MS components. In conclusion, from this study, it appears that $H I I T$ can be relevant for the prevention and treatment of the MS.
\end{abstract}

\section{Keywords}

Exercise, Metabolism, Obesity, Hyperglycemia, Diabetes Mellitus, Hypertension, Dyslipidemia.
Rev Bras Ativ Fis Saúde p. 669-687 DOI:

http://dx.doi.org/10.12820/rbafs.v.18n6p669

${ }^{1}$ Universidade Federal de Pelotas 


\section{INTRODUÇÃO}

A síndrome metabólica (SM) é conceituada pela associação de diversos fatores de riscos metabólicos, como diabetes mellitus tipo 2 (DM2), hipertensão arterial sistêmica (HAS), obesidade abdominal e dislipidemias (níveis elevados de triglicerídeos (TG) e baixos níveis de HDL - colesterol), além da presença de resistência à insulina $(\mathrm{RI})^{1}$.

Acerca de seu diagnóstico, há diferenças entre as entidades de saúde no estabelecimento de critérios para a identificação da $\mathrm{SM}^{1-3}$. Com o intuito de estabelecer um critério padronizado, uma normatização foi constituída em 2004 com a participação de diversas organizações de saúde ${ }^{3}$. Assim, atualmente, diagnostica-se SM a partir da presença de, pelo menos, três dos seguintes componentes: i) Circunferência abdominal aumentada, a partir de análise dos padrões da população estudada; ii) Pressão arterial $\geq 130 / 85 \mathrm{mmHg}$; iii) Glicemia de jejum $\geq 100 \mathrm{mg} / \mathrm{dL}$ ou estar em tratamento anti-hiperglicemiante; iv) Triglicerídeos $\geq 150 \mathrm{mg} / \mathrm{dL}$ ou estar em tratamento para dislipidemias; v) Colesterol HDL $<40 \mathrm{mg} / \mathrm{dL}$ (homens) e $<50 \mathrm{mg} / \mathrm{dL}$ (mulheres) ou estar em tratamento para dislipidemias ${ }^{3}$.

A prevalência mundial da SM é incerta e existem poucos estudos com tamanho amostral adequado para estimativas nacionais. Comparando resultados encontrados entre os anos de 1988 e 1994 na população estadunidense com pesquisas realizadas entre 1999 e 2000, verificou-se aumento na prevalência da SM de 50 para 64 milhões na população acima de 20 anos e, especialmente, no sexo feminino ${ }^{4}$. No mesmo estudo, considerando a falta de padronização entre os valores de referência para classificar a SM, os autores observaram que utilizando o conceito do National Cholesterol Education Program (NCEP) em 3.601 adultos, a prevalência de SM foi de 34,5\%, enquanto que, com os critérios do International Diabetes Federation (IDF), esta prevalência foi de 39\%. Em ambas as classificações foram encontradas prevalências superiores entre os homens ${ }^{4}$.

Considerando que o exercício físico é uma forma de terapia não-medicamentosa para a prevenção e o tratamento da SM, o objetivo da presente revisão é apresentar os diferentes estudos que fizeram uso desta estratégia e seus principais achados, com foco na aplicação do exercício intermitente de alta intensidade (HIIT).

\section{MATERIAS E MÉTODOS}

Para a organização desta revisão de literatura foram acessadas diferentes bases de dados - Pubmed/Medline, Scielo, HighWire, Google Scholar, com busca de artigos sem limite de datas. De modo complementar, as referências relevantes citadas pelos textos foram lidas e incorporadas à versão final. Para a busca, consideraram-se os idiomas português e inglês. Caso algum artigo em outro idioma fosse localizado, ele era incluído no processo de leitura. Como palavras-chave, foram empregados os unitermos "exercício intermitente", "exercício intervalado" e "exercício de alta intensidade", alternadamente combinados com o descritor "síndrome metabólica" nos dois idiomas usados. Para a sessão "Resultados de intervenções com exercícios físicos" foram incorporados dados decorrentes de exercícios contínuos e resistidos. Para as demais sessões, foram considerados os HIIT protocolos compostos por séries de esforços de curta ou média duração (6 s a 5 min) em intensidade acima do limiar anaeróbio, seguidos de períodos em baixa intensidade ou recuperação passiva ${ }^{5}$. Quanto aos delineamentos, foram incluídos estudos de intervenção e, quando oportuno, investigações observacionais que verificaram a relação entre realização de exercícios intensos e SM. 


\section{RESULTADOS DE INTERVENÇÕES COM EXERCÍCIOS FÍSICOS}

Considerando intervenções baseadas na prática de exercício físico, Seligman et al. (2011) ${ }^{6}$ conduziram um estudo com 75 portadores de SM que foram aleatorizados em três grupos: i) intervenção de 10.000 passos diários, contabilizados por pedômetro; ii) prática de exercício físico supervisionado, três dias por semana, com intensidade vigorosa e aconselhamento para caminhada rápida nos dias restantes; iii) aconselhamento para prática de caminhada diária com duração de 1 hora. Todos os grupos receberam orientações nutricionais. Após 12 semanas houve modificação do perfil lipídico, redução de pressão arterial, albumina e glicose em todos os grupos, porém com magnitude superior no grupo que realizou exercícios supervisionados. Após 1 ano de acompanhamento, 64\% dos participantes deixaram de ter SM, no entanto não houve descrição estratificada dos grupos neste momento da avaliação, o que prejudica o entendimento de qual estratégia foi mais efetiva no tratamento da $\mathrm{SM}^{6}$.

Resultados semelhantes foram encontrados em um estudo multicêntrico italiano no qual 691 portadores de DM2 e SM foram designados para: 1) um grupo controle, que recebeu aconselhamento para prática de atividade física regular;2) um grupo intervenção, que consistia na prática de exercício físico progressivo aeróbio e de força, duas vezes por semana e com alta intensidade. Após 12 meses, o grupo intervenção apresentou ganhos de aptidão física, além de redução da pressão arterial, circunferência abdominal, glicemia, $\mathrm{RI}$ e hemoglobina glicada $\left(\mathrm{HbA}_{1 \mathrm{c}}\right.$ - determina risco para DM2). Os autores ressaltaram que ofertar programas de exercício físico de alta intensidade e supervisionados pode ser uma estratégia adequada para a promoção de mudanças no estilo de vida de pacientes com o perfil apresentado ${ }^{7}$.

Com o intuito de investigar os efeitos do exercício físico após a redução de massa corporal e melhora do perfil metabólico de 102 indivíduos com SM, Thomas et al. $(2010)^{8}$ ofereceram aos voluntários um programa de exercício físico supervisionado associado a aconselhamento nutricional por um período de 4 a 6 meses, visando a redução de $10 \%$ na massa corporal. Na segunda fase do estudo, os voluntários foram randomizados em dois grupos: grupo "sem exercício físico", que continuou recebendo acompanhamento nutricional, e grupo "treinamento físico", que realizou a prática de exercício físico supervisionado em, no mínimo, três vezes por semana. Esta segunda fase tinha por objetivo auxiliar os sujeitos a recuperarem parcialmente a massa corporal de forma saudável e controlada. Durante a fase inicial, todos os marcadores da SM sofreram alterações importantes, principalmente relacionadas à redução da massa corporal. Porém, na segunda fase, as melhoras foram mantidas apenas no grupo que realizou treinamento físico. Neste sentido, este estudo é considerado o primeiro a demonstrar que os benefícios metabólicos podem ser mantidos mesmo quando há recuperação parcial de massa corporal perdida com a dieta ${ }^{8}$.

O Studies of a Targeted Risk Reduction Intervention through Defined Exercise (STRRIDE-AT/RT) foi a primeira investigação que objetivou comparar os efeitos do treinamento resistido isolado (TR), dos exercícios aeróbios (EA) e da combinação de ambos (TR-EA), nos componentes da SM. O grupo TR era realizado 3 vezes por semana em oito de exercícios de força com oito a doze repetições e o EA consistia de 120 min por semana em $75 \%$ do consumo máximo de oxigênio ( $\mathrm{VO}_{2}$ máx). Após oito meses, os grupos EA e TR-EA apresentaram redução da massa corporal, TG e circunferência abdominal, enquanto melhores índices de pressão arterial foram observados apenas no grupo TR-EA. Quanto ao grupo TR, não houve modificações em nenhum componente da SM${ }^{9}$. 
Também avaliando a combinação de prática de exercícios aeróbios e resistidos, Jurca et al., $(2004)^{10}$ registraram efeitos benéficos nos componentes lipídicos, glicêmicos e pressóricos e Mecca et al. (2012) ${ }^{11}$ relataram redução de $24 \%$ na prevalência de SM conseguida principalmente pela redução da circunferência abdominal.

Como o conceito de SM é relativamente recente, ainda é escasso o número de ensaios clínicos randomizados que analisaram os efeitos da prática de exercício físico supervisionado em indivíduos com todos os fatores simultaneamente. Porém, as revisões sistemáticas e metanálises apontam resultados positivos das intervenções baseadas em exercício físico, predominantemente aeróbios contínuos, na redução de glicemia de jejum, circunferência abdominal, pressão arterial e dislipidemia, o que pode diminuir o risco cardiovascular e a presença de $\mathrm{SM}^{19}$. Ressalta-se que a combinação de exercício físico com restrição na ingesta calórica promove melhorias superiores em todos os componentes da $\mathrm{SM}^{20-21}$.

Mesmo que a prática de exercícios aeróbios de intensidade moderada seja recomendada para promover modificações metabólicas e fisiológicas, observa-se um crescimento nos estudos baseados no conceito "time efficency", ou seja, a utilização de exercícios curtos com intensidade alta. Estes exercícios parecem: i) demonstrar ganhos expressivos de condicionamento físico, ii) gerar modificação da composição corporal a partir da diminuição da circunferência abdominal e do percentual de gordura e iii) proporcionar mudanças no perfil glicêmico e da $\mathrm{RI}^{22-24}$. Desta forma, é possível que estes exercícios sejam especialmente benéficos na SM.

\section{Exercício Intermitente de Alta Intensidade}

Algumas recomendações de atividade física para saúde se baseiam em exercícios contínuos de intensidade moderada que proporcionam, como visto anteriormente, aumento da potência aeróbia $\left(\mathrm{VO}_{2}\right.$ máx $)$ e redução e prevenção dos fatores de risco associadas à $\mathrm{SM}^{27}$.

No entanto, a associação de estímulos anaeróbios e aeróbios parece promover melhor controle metabólico que as atividades aeróbias isoladas ${ }^{66}$. Neste sentido, melhoras na sensibilidade insulínica (SI) estão mais relacionadas a exercício de alta intensidade e de baixo volume ${ }^{5}$. Neste contexto, os HIIT têm sido sugeridos como alternativa para promover maiores melhoras em menor tempo e aumentar a motivação e aderência aos programas de exercícios ${ }^{30}$.

Embora não haja textos especificamente voltados à classificação e conceituação do que é o HIIT, alguns trabalhos destacam que HIIT pode ser definido como exercícios de curta a moderada duração (10 s a $5 \mathrm{~min}$ ) realizados em intensidades superiores ao limiar anaeróbio/máxima fase estável do lactato ${ }^{5}$ e seguidos de pausas passivas ou ativas. Por outro lado, Gibala e McGee (2008) ${ }^{31}$ pontuam que o HIIT é o exercício com esforços repetidos na maior intensidade possível (all-out) ou próxima àquela do $\mathrm{VO}_{2 \max }\left(>90 \%\right.$ do $\mathrm{VO}_{2 \text { pico }}$ ). Assim, para melhor compreensão dos estudos com HIIT, as intensidades empregadas nos protocolos de treino serão sempre explicitadas.

\section{Respostas orgânicas agudas relacionadas ao HIIT}

Apesar de existirem dados consistentes sobre os efeitos decorrentes do treinamento de alta intensidade, informações sobre respostas agudas são relativamente limitadas ${ }^{32}$. De acordo com Boutcher $(2011)^{33}$, a partir das alterações imediatas e pós-esforço na frequência cardíaca (FC), hormônios, nível glicêmico, concentrações de lactato (LAC) e reatividade metabólica podem ser feitas inferências relacionadas aos fatores de risco associados à SM. Por exemplo, parece existir correlação entre obesidade, RI, disfunção no ventrículo esquerdo e disfunção diastólica 
e a $\mathrm{SM}^{34}$. Neste contexto, Ha et al. (2011) ${ }^{35}$ compararam a resposta de grupos de hipertensos com e sem SM (não diabéticos) a um teste incremental em cicloergômetro (25w a cada $3 \mathrm{~min}$ ) e concluíram que a SM influencia as funções sistólica e diastólica durante o exercício dinâmico, visto que a reserva contrátil, similar entre os grupos na situação de repouso, mostrou-se reduzida nos portadores da $\mathrm{SM}$ durante exercício. Da mesma forma, marcadores de disfunção diastólica, já presentes no repouso nos hipertensos com SM, aumentaram a cada estágio do protocolo de exercício, o que denota que esta disfunção é mantida durante esforço. A síntese dos efeitos agudos/imediatos do HIIT é apresentada no quadro 1.

Quadro 1 - Síntese dos efeitos agudos do HIIT.

\begin{tabular}{|c|c|c|c|c|}
\hline Estudo & $\begin{array}{l}\text { Características do } \\
\text { grupo }\end{array}$ & Tipo de treino & Intensidade do treino & Desfecho \\
\hline $\begin{array}{l}\text { Weltman et al. } \\
(2008)^{69}\end{array}$ & $\begin{array}{l}\text { Adultos obesos e não- } \\
\text { obesos }\end{array}$ & 1) $\begin{aligned} & \mathrm{HIIT}=3 \times \text { de } 10 \mathrm{~min} \\
& \times 10 \mathrm{~min} \\
& \text { 2) } \mathrm{EE}=30 \mathrm{~min}\end{aligned}$ & $\begin{array}{c}\text { HIIT = entre LL e } \\
\mathrm{VO}_{2} \text { pico } \\
\mathrm{EE}=\text { entre } \mathrm{LL} \mathrm{e} \mathrm{VO}_{2} \text { pico }\end{array}$ & $\begin{array}{l}\text { Resultados sugerem que ambos os } \\
\text { protocolos podem promover aumento na } \\
\text { secreção de GH medido } 24 \text { h pós-treino. }\end{array}$ \\
\hline $\begin{array}{l}\text { Ha et al. } \\
(2011)^{35}\end{array}$ & $\begin{array}{l}\text { Hipertensos com SM* } \\
\text { Hipertensos sem SM* }\end{array}$ & $\begin{array}{l}\text { Protocolo progresivo } \\
25 \mathrm{w} \text { a cada } 3 \mathrm{~min}\end{array}$ & $25 \mathrm{w}$ a cada $3 \mathrm{~min}$ & $\begin{array}{l}\text { SM é independentemente associada com } \\
\text { disfunção sistólica do ventrículo esquerdo } \\
\text { em pacientes hipertensos }\end{array}$ \\
\hline $\begin{array}{l}\text { Richards et al. } \\
(2010)^{47}\end{array}$ & $\begin{array}{l}\text { Homens, adultos } \\
\text { saudáveis }\end{array}$ & 4 séries de $30 \mathrm{~s} \times 4 \mathrm{~min}$ & All-out & $\begin{array}{l}\text { RI é afetada de maneira crônica, visto que não } \\
\text { há efeito evidenciado } 72 \mathrm{~h} \text { após sessão única. }\end{array}$ \\
\hline $\begin{array}{l}\text { Cristmass et al. } \\
(1999 b)^{39}\end{array}$ & $\begin{array}{l}\text { Homens, adultos } \\
\text { saudáveis }\end{array}$ & $\begin{array}{l}\text { 40min de exercício } \\
\text { intervalado: } \\
\text { 1) Curto: } 6 \mathrm{~s} \times 9 \mathrm{~s} \\
\text { 2) Longo: } 24 \mathrm{~s} \times 36 \mathrm{~s}\end{array}$ & $\begin{array}{l}\text { Razão de troca } \\
\text { respiratória } \\
\text { (de } 0,96 \text { a 0,99) }\end{array}$ & $\begin{array}{c}\text { Protocolo curto promoveu maiores taxas } \\
\text { de oxidação de gordura e de oxidação de } \\
\text { carboidratos. Essa diferença ocorreu devido } \\
\text { a fatores metabólicos relacionados a fibras } \\
\text { musculares oxidativas. }\end{array}$ \\
\hline $\begin{array}{l}\text { Cristmass et al. } \\
(1999 a)^{38}\end{array}$ & $\begin{array}{l}\text { Homens, adultos } \\
\text { saudáveis }\end{array}$ & $\begin{array}{l}\text { 90min contínuo } \\
90 \min 12 \mathrm{~s} \times 18 \mathrm{~s}\end{array}$ & $\begin{array}{l}60 \% \mathrm{VO}_{2} \text { pico } \\
120 \% \mathrm{VO}_{2} \text { pico }\end{array}$ & $\begin{array}{l}\text { Existe diferença na utilização dos substratos } \\
\text { entre os diferentes protocolos. Sendo que o } \\
\text { HIIT promoveu maior oxidação glicolítica e } \\
\text { oxidação de gordura } 3 \text { vezes menor que o } \\
\text { protocolo contínuo. }\end{array}$ \\
\hline $\begin{array}{l}\text { Mandroukas } \\
\text { et al. }(2011)^{40}\end{array}$ & $\begin{array}{l}\text { Homens, adultos } \\
\text { saudáveis }\end{array}$ & $\begin{array}{l}\text { 32min contínuo } \\
\text { 32min ( } 4 \text { min } x 4 \text { min) } \\
\text { Recuperação ativa } x \\
\text { pasiva }\end{array}$ & $\begin{array}{c}12 \mathrm{~km} / \mathrm{h} \\
12 \mathrm{~km} / \mathrm{h} \times 8 \mathrm{~km} / \mathrm{h}\end{array}$ & $\begin{array}{c}\text { O protocolo contínuo pareceu mais } \\
\text { eficiente na solicitação do componente } \\
\text { cardiovascular por induzir maior FCmáx e } \\
\text { maior } \mathrm{VO}_{2}\end{array}$ \\
\hline $\begin{array}{l}\text { Gillen et al. } \\
(2012)^{43}\end{array}$ & Portadores de DM2 & 10 sriesde $60 \mathrm{~s} \times 60 \mathrm{~s}$ & 90\% FCmáx & $\begin{array}{l}\text { Redução no tempo em hiperglicemia, menor } \\
\text { pico glicêmico pós-refeição, redução na } \\
\text { glicemia } 60-120 \text { min pós-refeição e redução } \\
\text { do tempo abaixo da curva glicêmica. }\end{array}$ \\
\hline $\begin{array}{l}\text { Gosselin et al. } \\
(2011)^{77}\end{array}$ & $\begin{array}{l}\text { Adultos jovens } \\
\text { fisicamente ativos }\end{array}$ & $\begin{array}{c}\mathrm{EE}=20 \mathrm{~min} \\
\text { HIIT }(\mathrm{s})= \\
30 \times 30 / 60 \times 30 / 90 \times 30 / \\
60 \times 60\end{array}$ & $\begin{array}{l}70 \% \mathrm{VO}_{2} \text { máx } \\
90 \% \mathrm{VO}_{2} \text { máx }\end{array}$ & $\begin{array}{l}\text { As demandas fisiológicas são maiores no } \\
\text { HIIT do que no EE, e as respostas do HIIT } \\
\text { dependem da razão esforço:pausa aplicada. }\end{array}$ \\
\hline $\begin{array}{l}\text { Goto et al. } \\
(2011)^{78}\end{array}$ & Homens saudáveis & $\begin{array}{l}1 \times 30 \mathrm{~min} \\
3 \times 10 \mathrm{~min}\end{array}$ & $60 \% \mathrm{VO}_{2}$ máx & $\begin{array}{c}\text { A característica intermitente contribui para } \\
\text { maior taxa de oxidação de gordura durante } \\
\text { exercício. }\end{array}$ \\
\hline
\end{tabular}

$\mathrm{SM}=$ Síndrome metabólica; DM2= Diabetes Mellitus tipo2; EE= Exercício de intensidade e duração que promova estado-estável; HIIT= High Intensity Intermitent Training (Exercício intermitente de alta intensidade); FCres= Frequência cardíaca de reserva; TM= Trabalho máximo; $M C=$ Massa corporal; $M G=$ Massa gorda; $\mathrm{RI}=$ Resistência insulínica; $\mathrm{GH}=$ Hormônio do crescimento; $\mathrm{LL}=$ Limiar de lactato; All-out= Esforços executados na maior intensidade possível. ${ }^{*}=$ Ambos grupos sem DM2.

\section{Respostas cardiovasculares}

Quanto ao componente cardiovascular, respostas agudas apontam que um estímulo único de 30 s all-out, apesar de apresentar demanda de aproximadamente $90 \%$ da FCmax e do $\mathrm{VO}_{2}$ pico, não aumenta a capacidade máxima de ejeção cardíaca ${ }^{32}$. 
Porém, este esforço implica na contribuição de 17\% do sistema ATP-CP, 45\% do sistema glicolítico e $38 \%$ do metabolismo oxidativo ${ }^{36}$. Assim, infere-se que a melhora no desempenho físico advinda do treinamento com estas características corresponda a ganhos periféricos, pois para se obter melhoras centrais é necessária uma maior quantidade de esforço e/ou estímulos mais longos, superiores a $1 \mathrm{~min}^{32}$. Com isso, a partir da soma dos estímulos, ainda considerando $30 \mathrm{~s}$ de atividade all -out, a demanda aeróbia é aumentada gradativamente a cada esforço subsequente, sendo que na terceira série de $30 \mathrm{~s}$, com recuperação de 4 min entre cada uma, a contribuição aeróbia chega a $70 \%{ }^{37}$.

Ainda considerando a demanda energética dos estímulos, Cristmass et al. $(1999)^{38}$, ao compararem protocolo submáximo contínuo (90min a $60 \%$ do $\mathrm{VO}_{2}$ pico) com protocolo intermitente de alta intensidade (90min, 12 s a $120 \% \mathrm{VO}_{2}$ pico e 18s em repouso), identificaram que a oxidação de gordura é três vezes maior no primeiro e a oxidação glicolítica é predominante no segundo protocolo, apesar de $\mathrm{VO}_{2}$ e gasto energético semelhantes. A mesma proporção metabólica foi encontrada na comparação de 40 min de exercício intermitente curto $(6 \times 9$ s) e longo ( $24 \times 36$ s), com intensidade de esforço determinada a partir da razão de troca respiratória (entre 0,96 e 0,99$).{ }^{39}$.

Outro aspecto é que por apresentar maior solicitação aeróbia, corridas de 32 min parecem ser mais eficientes na solicitação do sistema cardiovascular (maior FC e maior $\mathrm{VO}_{2}$ médios durante a sessão) se executadas de maneira contínua $(12 \mathrm{~km} / \mathrm{h})$ que quando comparadas ao mesmo tempo de corrida realizado de maneira intervalada $(4 \mathrm{~min}$ a $12 \mathrm{~km} / \mathrm{h}$ x $4 \mathrm{~min}$ a $8 \mathrm{~km} / \mathrm{h}$ ), independentemente do tipo de recuperação aplicada, ativa com $4 \mathrm{~min}$ a $8 \mathrm{~km} / \mathrm{h}$ ou passiva ${ }^{40}$.

Analisados os dados anteriores em conjunto, pode parecer mais vantajoso fazer o exercício contínuo, mas não o é. A intolerância ao exercício e a possibilidade de desistência durante a sessão de treino aumentam consideravelmente quando tal estratégia é adotada. Ao se realizar exercícios contínuos em cicloergômetro na potência máxima ( 100 a $102 \%$ da potência do $\mathrm{VO}_{2 \max }$ ), registram-se durações de 4 a $6 \mathrm{~min}$; por outro lado, ao se intervalar esforços e pausas de $15 \mathrm{~s}$, o exercício pode ser mantido por até uma hora ${ }^{41}$.

Currie et al. (2012) ${ }^{42}$ realizaram uma investigação acerca das alterações na função endotelial em dez indivíduos com doença arterial coronariana. Para isto, aplicaram-se uma sessão de treino de intensidade moderada (30min a 55\% do $\mathrm{VO}_{2}$ pico) e uma de HIIT (dez séries de $1 \mathrm{~min}$ a $80 \%$ do $\mathrm{VO}_{2}$ pico com intervalos de $1 \mathrm{~min}$ a $10 \%$ do $\mathrm{VO}_{2}$ pico). O trabalho total foi maior nos exercícios moderados $(166 \pm 52 \mathrm{~kJ})$ em relação ao HIIT $(93 \pm 28 \mathrm{~kJ})$; porém não foram encontradas diferenças nas respostas agudas da função endotelial ${ }^{42}$.

\section{Resposta glicêmica e insulínica}

A hiperglicemia pós-refeição é considerada o principal fator agravante das complicações relacionadas ao DM2, incluindo as DCV. Gillen et al. (2012) ${ }^{43}$, ao monitorarem o comportamento do nível glicêmico de indivíduos com DM2 durante 24h após uma sessão de 10 min de HIIT (10x60s em 90\% da FCmax por 60 s de recuperação) e compararem com grupo que não se exercitou, evidenciaram valores menores do tempo em hiperglicemia ( $>10 \mathrm{mmol} / \mathrm{L}$ ), do pico glicêmico pós-refeição, na glicemia entre 60 e 120 min pós-refeição e da área sob a curva glicêmica por $3 \mathrm{~h}$. Assim, o referido protocolo, com duração total de 20 min, constitui-se como uma estratégia prática e temporalmente eficiente para o controle glicêmico nesta população. Apesar das recomendações de $2,5 \mathrm{~h} / \mathrm{sem}$ de exercícios aeróbios para prevenir a progressão 
da $\mathrm{DM} 2{ }^{44}$, e de 4-7 h/sem para a manutenção de massa corporal saudável ${ }^{14}$, tem sido sugerido que sessões curtas de 10-20 min de HIIT podem manter a SI no dia do exercício. Neste caso, a característica intermitente, que tem mostrado efetividade na redução da concentração de insulina de jejum, pode auxiliar indivíduos pouco condicionados a cumprirem suas metas diárias ${ }^{45}$.

Whyte et al. (2012) ${ }^{46}$ investigaram estímulos de alta intensidade executados de maneira intermitente ( 4 × $30 \mathrm{~s}$ all-out por 4,5 min de recuperação) ou em um único sprint máximo até que fosse atingido mesmo trabalho total do protocolo intermitente. Estes autores concluíram, a partir de coletas realizadas no dia seguinte, que os dois protocolos foram capazes de promover aumento da oxidação de gordura (intermitente: $63 \%$ vs sprint único: $38 \%$ ), porém, o sprint único apresentou tempo total menor (20min vs 3,3min). Os autores especularam que treino com sprint único pode ser uma ferramenta temporalmente eficiente, já que mostrou aumento da SI e da oxidação de gordura em homens adultos com sobrepeso e obesos.

Por outro lado, Richards et al. (2010) ${ }^{47}$ reportaram não haver resposta significante de aumento da SI 72h após sessão única de HIIT $(4$ x 30 s all-out por 4 min de recuperação) e concluíram que o aumento evidenciado a partir de seis sessões do mesmo protocolo foi decorrente do impacto crônico do treinamento.

\section{Obesidade}

De maneira aguda, a mobilização da gordura visceral abdominal está mais associada a exercícios de maior intensidade que induzem secreção de hormônios lipolíticos $(\mathrm{GH})$, aumentam o gasto energético pós-exercício e elevam a oxidação da gordura fornecendo um maior balanço energético negativo ${ }^{48-51}$. Tsekouras et al. $(2008)^{52}$ evidenciaram que a secreção de lipoproteínas de muito baixa densidade (VLDL) e TG apresenta redução significativa 48 h pós-treino. Neste mesmo estudo, os autores afirmaram que existe aumento na disponibilidade de AGL no plasma sanguíneo $24 \mathrm{~h}$ pós-treino, e estas alterações são favoráveis ao metabolismo lipídico, que está associado à redução de fatores risco de doença coronariana e aterosclerose ${ }^{52}$.

\section{Respostas orgânicas crônicas relacionadas ao HIIIT}

De acordo com Boutcher (2010) $)^{33}$, as respostas crônicas estão relacionadas às modificações na aptidão aeróbia e anaeróbia, adaptações musculares, redução dos níveis de insulina de jejum e SI que ocorrem após um período de treinamento. Apesar de a maior parte dos estudos que investigaram os efeitos do HIIT de maneira crônica ser baseada em períodos curtos de treinamento ( 2 a 6 semanas), existem evidências dos efeitos após $15^{50,53}$ e até mesmo 32 semanas $^{54}$. A síntese de diferentes respostas crônicas do HIIT é apresentada no quadro 2.

\section{Respostas Cardiovasculares}

Considerando o risco cardiovascular aumentado de indivíduos com SM, é importante ressaltar que elevações na aptidão cardiorrespiratória estão associadas à redução de risco, sendo que a intensidade é um fator determinante na indução de melhora nesta variável. Neste sentido um treinamento de alta intensidade $(3 \mathrm{~d} / \mathrm{sem}$ acima do limiar de lactato (LL) e $2 \mathrm{~d} /$ sem abaixo do LL) promoveu ganhos superiores na aptidão cardiorrespiratória ( $14 \%$ vs $9 \% \mathrm{VO}_{2}$ pico) do que os induzidos por intensidades menores ( $5 \mathrm{~d} /$ sem abaixo do LL), após 16 semanas de intervenção, estando ambos os protocolos associados à redução da pressão arterial de repouso ${ }^{49}$.

Adicionalmente, estímulos de sprints promovem adaptações orgânicas positivas, que possibilitam a manutenção do desempenho em alta intensidade. Dentre estas, 
Quadro 2 - Síntese dos efeitos orgânicos crônicos do HIIT.

\begin{tabular}{|c|c|c|c|c|}
\hline Estudo & $\begin{array}{c}\text { Características } \\
\text { do grupo }\end{array}$ & Tipo de treino & Intensidade do treino & Desfecho \\
\hline $\begin{array}{l}\text { Gutin et al. } \\
(2002)^{54}\end{array}$ & $\begin{array}{c}\text { Adolescentes } \\
\text { obesos }\end{array}$ & $\begin{array}{l}\text { Alta intensidade } \\
\text { Baixa intensidade } \\
8 \text { Meses }\end{array}$ & $\begin{array}{l}75 \text { a } 80 \% \mathrm{VO}_{2} \text { pico } \\
50 \text { a } 60 \% \mathrm{VO}_{2} \text { pico }\end{array}$ & $\begin{array}{l}\text { Melhor desempenho no } \mathrm{VO}_{2} \text { máx, } \\
\text { especialmente a partir do protocolo } \\
\text { de maior intensidade, e redução da } \\
\text { gordura visceral e total, independente } \\
\text { da intensidade. }\end{array}$ \\
\hline $\begin{array}{l}\text { Irving et al. } \\
(2009)^{51}\end{array}$ & $\begin{array}{c}\text { Adultos com } \\
\text { SM }\end{array}$ & $\begin{array}{c}\text { 3d/sem HIIT }+2 d / \text { sem EE } \\
5 d / \text { sem EE } \\
16 \text { Semanas }\end{array}$ & $\begin{array}{l}3 d / \text { sem acima do } L L \\
2 d / \text { sem abaixo do } L L \\
5 d / \text { sem abaixo do } L L\end{array}$ & $\begin{array}{l}\text { Foi evidenciado aumento superior } \\
\text { da secreção noturna de GH a } \\
\text { partir do HIIT. Adicionalmente, } \\
\text { alterações favoráveis na composição } \\
\text { corporal foram identificadas, } \\
\text { independentemente da intensidade. }\end{array}$ \\
\hline
\end{tabular}

HIIT curto $=10$ a 15 sprints de 10 a $15 \mathrm{~s} \times$ recuperação até atingir $120 \mathrm{a}$ $130 \mathrm{bpm}$

Tremblay et HIIT longo $=5$ a 6 sprints de 60 a 90 al. $(1994)^{53} \quad$ Adultos jovens $s \times$ recuperação até atingir 120 a 130 bpm

15 Semanas

$\mathrm{EE}=30$ a 45min durante 20 Semanas
$60 \%$ do TM em 10 seg $70 \%$ do TM em 90seg $60 \%$ a $85 \%$ da FCres
Protocolos intermitentes promoveram maior redução da gordura subcutânea através do favorecimento dos processos de oxidação lipídica.

\begin{tabular}{|c|c|c|c|c|}
\hline $\begin{array}{l}\text { Babraj et al. } \\
(2009)^{30}\end{array}$ & $\begin{array}{l}\text { Adultos jovens } \\
\text { sedentários }\end{array}$ & $\begin{array}{c}6 \text { sessões de } 4-6 \text { séries de Wingate } \\
\text { (30s } \times 4 \mathrm{~min}) \\
2 \text { Semanas }\end{array}$ & All-out & $\begin{array}{l}\text { Ação insulínica e homeostase da } \\
\text { glicemia foram otimizadas em função } \\
\text { do alto volume de massa muscular } \\
\text { envolvida e pela alta taxa de quebra e } \\
\text { ressíntese de glicogênio derivadas da } \\
\text { alta intensidade. }\end{array}$ \\
\hline $\begin{array}{l}\text { Moghadasi } \\
\text { et al. } \\
(2011)^{63}\end{array}$ & $\begin{array}{l}\text { Adultos com } \\
\text { sobrepeso e } \\
\text { obesos }\end{array}$ & 45min Contínuo por 12 Semanas & $80 \% \mathrm{VO}_{2} \max$ & $\begin{array}{l}\text { Alta intensidade mostrou elevação dos } \\
\text { níveis de adiponectina e redução da RI, } \\
\text { relacionados a aumento da expressão } \\
\text { do RNAm em adultos obesos. }\end{array}$ \\
\hline $\begin{array}{l}\text { Metcalfe et } \\
\text { al. }(2011)^{64}\end{array}$ & $\begin{array}{l}\text { Adultos } \\
\text { saudáveis e } \\
\text { sedentários }\end{array}$ & $\begin{array}{c}\text { Semanas } 2-3 \text { - } 2 \text { séries de } 10 \mathrm{~s} \times 3 \mathrm{~min} \\
\text { e } 40 \mathrm{~s} \\
\text { Semanas } 4-9-2 \text { séries de } 15 \mathrm{~s} \times 3 \mathrm{~min} \\
\text { e } 30 \mathrm{~s} \\
\text { Semanas } 10-18-2 \text { séries de } 20 \mathrm{~s} \times \\
3 \text { min e } 20 \mathrm{~s} \\
\text { Total de } 18 \text { Semanas }\end{array}$ & All-out & $\begin{array}{l}\text { O protocolo de HIIT reduzido foi } \\
\text { suficiente para promover aumento da } \\
\text { SI e do } \mathrm{VO}_{2} \text { pico. }\end{array}$ \\
\hline $\begin{array}{l}\text { Andrews et } \\
\text { al. }(2011)^{28}\end{array}$ & $\begin{array}{l}\text { Homens } \\
\text { adultos com } \\
\text { DM2 }\end{array}$ & $\begin{array}{l}\text { 30min de caminhada } 5 \mathrm{~d} / \text { sem durante } \\
\qquad 12 \text { Meses }\end{array}$ & Moderada & $\begin{array}{l}\text { Não houve diferença na resposta } \\
\text { glicêmica a partir do protocolo de } \\
\text { exercícios devido a intensidade } \\
\text { ser insuficiente para promover tal } \\
\text { adaptação. }\end{array}$ \\
\hline $\begin{array}{l}\text { Litlle et al. } \\
(2011)^{62}\end{array}$ & $\begin{array}{l}\text { Homens } \\
\text { adultos com } \\
\text { DM2 }\end{array}$ & $\begin{array}{c}6 \text { sessões de } 10 \text { séries de } 60 \mathrm{~s} \times 60 \mathrm{~s} \\
\text { durante } 2 \text { Semanas }\end{array}$ & $90 \%$ FCmax & $\begin{array}{l}\text { Treinamento intenso é eficiente para } \\
\text { melhorar controle glicêmico em } \\
\text { pacientes com DM2, visto a redução } \\
\text { da glicemia } 24 \text { pós-treino, aumento da } \\
\text { capacidade mitocondrial e GLUT4. }\end{array}$ \\
\hline $\begin{array}{l}\text { Tsekouras et } \\
\text { al. }(2008)^{52}\end{array}$ & $\begin{array}{l}\text { Homens } \\
\text { adultos } \\
\text { sedentários }\end{array}$ & $\begin{array}{c}3 \mathrm{~d} / \mathrm{sem}, 32 \mathrm{~min}=4 \mathrm{~min} \times 4 \mathrm{~min} \\
\text { durante } 2 \text { Meses }\end{array}$ & $\begin{array}{l}4 \min \text { a } 60 \% \text { e } \\
4 \text { min a } 90 \% \text { do } \\
\mathrm{VO}_{2} \text { máx }\end{array}$ & $\begin{array}{l}\text { Foi evidenciada redução nas taxas } \\
\text { de VLDL e TG tanto em jejum } \\
\text { quanto na taxa de secreção hepática. } \\
\text { Estas adaptações podem diminuir } \\
\text { o risco de esteatose hepática e } \\
\text { hipertrigliceridemias. }\end{array}$ \\
\hline
\end{tabular}

Continua... 
... continua

\begin{tabular}{|c|c|c|c|c|}
\hline Estudo & $\begin{array}{l}\text { Características } \\
\text { do grupo }\end{array}$ & Tipo de treino & Intensidade do treino & Desfecho \\
\hline $\begin{array}{l}\text { Astorino et } \\
\text { al. }(2011)^{57}\end{array}$ & Jovens ativos & $\begin{array}{c}6 \text { sessões de séries de Wingate } \\
\text { (30s } \times 4 \text { min), durante } 2 \text { semanas } \\
4 \times \text { nos dias } 1 \text { e } 2 / 5 \text { nos dias } 3 \text { e } 4 / \\
6 \times \text { nos dias } 5 \text { e } 6\end{array}$ & All-out & $\begin{array}{l}\text { Incremento no } \mathrm{VO}_{2} \text { máx e na potência } \\
\text { de ejeção cardíaca a partir da melhora } \\
\text { na função cardíaca e na captação de } \\
\qquad \mathrm{O}_{2} .\end{array}$ \\
\hline $\begin{array}{l}\text { Trilk et al. } \\
(2011)^{55}\end{array}$ & $\begin{array}{l}\text { Mulheres com } \\
\text { sobrepeso/ } \\
\text { obesas e } \\
\text { sedentárias }\end{array}$ & $\begin{array}{l}\text { 4-7 séries de } 30 \text { s all-out } \times 4 \mathrm{~min} \text {, } \\
\text { durante } 4 \text { semanas }\end{array}$ & $\begin{array}{l}\text { Acima de } 240 \% \\
\text { da intensidade do } \\
\mathrm{VO}_{2} \text { máx }\end{array}$ & $\begin{array}{l}\text { Aumento do volume de ejeção pelo } \\
\text { ventrículo esquerdo, aumento do } \\
\text { VO }_{2} \text { máx e redução da FCrep. }\end{array}$ \\
\hline $\begin{array}{l}\text { Trapp et al. } \\
(2008)^{50}\end{array}$ & $\begin{array}{l}\text { Mulheres } \\
\text { jovens e } \\
\text { sedentárias }\end{array}$ & $\begin{array}{l}\text { 1) } \mathrm{HIIT}=60 \text { séries de } 8 \mathrm{~s} \times 12 \mathrm{~s} \\
\text { 2) } \mathrm{EE}=20 \mathrm{~min} \text { a } 40 \mathrm{~min} \\
15 \text { Semanas }\end{array}$ & $\begin{array}{l}\text { All-out } \\
60 \% \mathrm{VO}_{2} \text { pico }\end{array}$ & $\begin{array}{c}\text { Aumento na capacidade aeróbia } \\
\text { absoluta e redução da insulina de } \\
\text { jejum, na gordura corporal total, } \\
\text { gordura subcutânea da coxa e gordura } \\
\text { abdominal a partir do HIIT. }\end{array}$ \\
\hline $\begin{array}{l}\text { Irving et al. } \\
(2008)^{49}\end{array}$ & $\begin{array}{l}\text { Mulheres } \\
\text { obesas com } \\
\text { SM }\end{array}$ & $\begin{array}{c}\text { 3d/sem HIIT e } 2 d / \text { sem EE } \\
5 d / \text { sem EE } \\
16 \text { Semanas }\end{array}$ & $\begin{array}{l}3 d / \text { sem acima do } L L \\
2 d / \text { sem abaixo do } L L \\
5 d / \text { sem abaixo do } L L\end{array}$ & $\begin{array}{l}\text { Maior ganho de potência aeróbia e } \\
\text { maior redução da gordura abdominal } \\
\text { total, da gordura subcutânea } \\
\text { abdominal e da gordura visceral } \\
\text { abdominal a partir do protocolo } \\
\text { intenso. }\end{array}$ \\
\hline $\begin{array}{l}\text { Freyssin et } \\
\text { al. }(2012)^{56}\end{array}$ & $\begin{array}{l}\text { Pacientes com } \\
\text { falha cardíaca } \\
\text { crônica }\end{array}$ & $\begin{array}{l}\mathrm{HIIT}=3 \text { séries, com intervalo de } 5 \mathrm{~min} \\
\text { entre elas, de } 12 \text { sprints de } 30 \mathrm{~s} \times 60 \mathrm{~s} \\
\mathrm{EE}=45 \mathrm{~min} \\
8 \text { Semanas }\end{array}$ & $\begin{array}{c}80 \% \mathrm{VO}_{2} \text { máx } \\
\text { Limiar ventilatório } 1\end{array}$ & $\begin{array}{l}\text { HIIT promoveu ganhos no } \mathrm{VO}_{2} \text { pico, } \\
\text { teste de duração de exercício, captação } \\
\text { de } \mathrm{O}_{2} \text { durante exercício máximo e } \\
\text { no } \mathrm{VO}_{2} \text { consumido na velocidade do } \\
\text { limiar ventilatório } 1 \text {. O mesmo não foi } \\
\text { evidenciado com o protocolo contínuo. }\end{array}$ \\
\hline $\begin{array}{l}\text { Tabata et al. } \\
(1996)^{80}\end{array}$ & $\begin{array}{l}\text { Homens } \\
\text { adultos jovens } \\
\text { universitários }\end{array}$ & $\begin{array}{l}\text { 7-8 séries } 20 \mathrm{~s} \times 10 \mathrm{~s} \\
6 \text { Semanas }\end{array}$ & $170 \% \mathrm{VO}_{2}$ máx & $\begin{array}{l}\text { HIIT prescrito de maneira adequada } \\
\text { pode promover adaptações nos } \\
\text { sistemas aeróbio e anaeróbio, } \\
\text { provavelmente, pela alta intensidade } \\
\text { imposta em ambos os sistemas. }\end{array}$ \\
\hline $\begin{array}{l}\text { Hood et al. } \\
(2011)^{81}\end{array}$ & $\begin{array}{l}\text { Adultos jovens } \\
\text { sedentarios }\end{array}$ & $\begin{array}{l}10 \times 1 \min \times 1 \min \\
2 \text { Semanas }\end{array}$ & $80-95 \%$ FCres & $\begin{array}{l}\text { O protocolo proposto induziu } \\
\text { rapidamente adaptações na biogênese } \\
\text { mitocondrial musculoesquelética, } \\
\text { aumento de GLUT4 e melhora na } \\
\text { sensibilidade a insulina e representa } \\
\text { alternativa temporalmente eficiente } \\
\text { para redução de riscos metabólicos a } \\
\text { saúde. }\end{array}$ \\
\hline
\end{tabular}

$\mathrm{SM}=$ Síndrome metabólica; $\mathrm{DM} 2=$ Diabetes Mellitus tipo2; EE= Exercício de intensidade e duração que promova estado-estável; HIIT= High Intensity Intermittent Training (Exercício intermitente de alta intensidade); FCmáx= Frequência cardíaca máxima; FCres= Frequência cardíaca de reserva; FCrep= Frequência cardíaca de repouso; $T M=$ Trabalho máximo; $M C=$ Massa corporal; $M G=$ Massa gorda; $\mathrm{RI}=\mathrm{Resistência} \mathrm{insulí-}$ nica; LL= Limiar de lactato; VO2máx= Consumo máximo de oxigênio; TG= Triglicerídeos; VLDL= lipoproteína de muito baixa densidade; GH= Hormônio do crescimento; All-out= Esforços executados na maior intensidade possível.

são evidenciadas melhora da distensibilidade arterial, da função endotelial, da capacidade oxidativa muscular e dos estoques de glicogênio ${ }^{32}$. Freyssin et al. $(2012)^{56}$ observaram resultados consideravelmente superiores em pacientes com insuficiência cardíaca crônica com HIIT ( 3 séries, com intervalos de 5 min entre elas, de 12 sprints de 30 s em $80 \%$ da potência máxima x 60 s de recuperação passiva) quando comparado ao exercício contínuo ( $45 \mathrm{~min}$ na velocidade do limiar ventilatório1). O grupo HIIT apresentou ganhos no $\mathrm{VO}_{2}$ pico (27\%), na duração do exercício (47\%), na captação de $\mathrm{O}_{2}$ durante exercício máximo (18\%) e no $\mathrm{VO}_{2}$ consumido na velocidade do limiar ventilatório1 (22\%), o que não foi observado no protocolo de treino contínuo ${ }^{56}$. 
Ainda considerando a função cardíaca, o treinamento em intensidades supramáximas ( $>240 \%$ da intensidade do $\mathrm{VO}_{2} \max$ ) aumentaram o volume de ejeção do ventrículo esquerdo e o $\mathrm{VO}_{2}$ máx e reduziram a $\mathrm{FCrep}^{55}$. Este protocolo, de 4-7 séries de sprints de $30 \mathrm{~s}$ all-out, seguidos de 4 min de recuperação passiva, mostrou-se adequado para o aprimoramento do componente aeróbio central em mulheres com sobrepeso/obesas sedentárias após quatro semanas de prática. Os autores concluíram, portanto, que exercícios intensos podem promover ganhos cardiovasculares importantes para a manutenção de saúde e redução do risco de mortalidade por doenças crônicas relacionadas à obesidade e o sedentarismo, em aproximadamente metade do período descrito para exercícios contínuos de maior volume e menor intensidade ${ }^{55}$. Corroborando com estes achados, Trapp et al. $(2008)^{50}$ encontraram aumento superior da capacidade aeróbia com HIIT quando comparado ao exercício contínuo moderado (23 vs 19\% no $\mathrm{VO}_{2}$ pico), mesmo com o volume de treinamento aeróbio sendo maior no segundo grupo (36 vs $120 \mathrm{~min} / \mathrm{sem}$ ).

Gutin et al. (2002) $)^{54}$ associaram aulas sobre estilo de vida saudável a exercícios físicos por oito meses e encontraram melhora do $\mathrm{VO}_{2}$ max de adolescentes obesos com protocolo de maior intensidade (75 a $80 \% \mathrm{VO}_{2}$ pico). Além disso, esta melhor resposta foi positivamente correlacionada ao tempo gasto em atividades vigorosas, o mesmo não aconteceu no grupo que executou exercícios em intensidade moderada (50 a 60\% $\mathrm{VO}_{2}$ pico). Em outra investigação, Astorino et al. (2011) ${ }^{57}$ aplicaram um protocolo com testes de Wingate (30s all-out $\mathrm{x} 4 \mathrm{~min}$ de recuperação passiva) durante 6 sessões ( $4 \mathrm{x}$ nas sessões 1 e 2 ; $5 \mathrm{x}$ nas sessões 3 e 4; $6 \mathrm{x}$ nas sessões 5 e 6) em jovens ativos e identificaram aumento no $\mathrm{VO}_{2} \max$ e na potência de ejeção cardíaca, o que reflete uma melhora na função cardíaca e na captação de $\mathrm{O}_{2}$. Morikawa et al. (2011) $)^{58}$ avaliaram os efeitos do treinamento de caminhadas intervaladas no autorrelato de doenças em 666 idosos japoneses. Os participantes foram divididos em três grupos distintos conforme o $\mathrm{VO}_{2}$ max inicial. $\mathrm{O}$ protocolo de exercício consistiu em cinco séries de 3 min de caminhada lenta $\left(40 \% \mathrm{VO}_{2}\right.$ pico) seguidos por 3 min de caminhada rápida (entre $70-80 \% \mathrm{VO}_{2}$ pico) em $\geq 4$ dias/ sem. Após quatro meses, o treinamento de caminhada intermitente aumentou o $\mathrm{VO}_{2}$ pico e reduziu a incidência de doenças relacionadas ao estilo de vida. Adicionalmente, a incidência de doenças cardíacas foi maior entre o grupo de $\mathrm{VO}_{2}$ pico mais baixo que os demais, uma tendência semelhante foi observada para as variáveis hipertensão e uso de medicamentos para hiperglicemia.

Ainda considerando a população de idosos, o declínio do $\mathrm{VO}_{2}$ pico, $30 \%$ a cada década após os $30 \operatorname{anos}^{59}$, mostrou-se amenizado com duas sessões semanais de HIIT durante 9 semanas. O protocolo de $30 \mathrm{~min}$ (6x de $4 \mathrm{~min}$ no limiar ventilarório1 alternados com $1 \mathrm{~min}$ no limiar ventilatório2) aumentou o $\mathrm{VO}_{2}$ pico em $15 \%$ em homens e mulheres maiores de 60 anos. Segundo os autores, as adaptações se relacionaram ao decréscimo da quebra de glicogênio e ao aumento da solicitação da via oxidativa decorrente do treinamento, o que gerou melhora no transporte e na captação de $\mathrm{O}_{2}{ }^{60}$.

\section{Resposta glicêmica e insulínica}

Em um estudo, Andrews et al. $(2011)^{28}$ reportaram não haver diferença na resposta glicêmica após o tratamento com dieta e a combinação da dieta com a atividade física em homens adultos com DM2. Os autores propuseram que o protocolo de exercícios utilizado (exercícios moderados em 5 dias/sem) teve intensidade insuficiente e que a associação entre exercícios aeróbios e anaeróbios poderia melhorar o controle metabólico. Esta suposição vai ao encontro dos achados produzidos por 
Trapp et al. $(2008)^{50}$, que evidenciaram redução de $31 \%$ na insulina plasmática de jejum após 15 semanas de HIIT, enquanto exercícios moderados apresentaram resultado modesto ( $9 \%$ de redução). Assim, os autores sugeriram o HIIT como uma forma eficiente de exercício para a normalização de disfunções endócrinas e, em longo prazo, a redução da concentração de insulina pode aumentar a oxidação de gordura e reduzir a massa corporal. Há ainda indícios de que 4 meses de caminhadas intervaladas de alta intensidade resultem em maiores melhoras no controle glicêmico, redução da massa e da gordura corporal e ganho no $\mathrm{VO}_{2} \max$ em pessoas com DM do que a caminhada contínua de intensidade moderada ${ }^{61}$.

Os efeitos do exercício aeróbio sobre a ação da insulina parecem ser independentes da resposta na composição corporal e a melhora na RI parece estar relacionada a esforços de alta intensidade. $\mathrm{O}$ músculo esquelético apresenta grande atuação na captação de glicose, assim, apenas alguns minutos de exercício intervalado de alta intensidade ( 6 sessões de $15 \mathrm{~min}$ em duas semanas, 4- 6 a séries de Wingate) podem otimizar substancialmente a ação insulínica e a homeostase da glicose em adultos jovens sedentários em função do alto volume de massa muscular envolvida e pela alta taxa de quebra e ressíntese de glicogênio derivada da alta intensidade ${ }^{30}$. Complementarmente, Little et al. $(2011)^{62}$ aplicaram seis sessões de HIIT (10x60s a 90\% FCmáx x 60s recuperação) em duas semanas em adultos com DM2 e evidenciaram redução da média da glicemia 24 h (7,6 vs 6,6 $\mathrm{mmol} / \mathrm{L}$ ), aumento da capacidade mitocondrial e GLUT4 muscular (principal transportador da glicose no músculo esquelético) em aproximadamente $369 \%$. De forma semelhante, outro estudo observou que 6 sessões de treinos ( 8 a 12 esforços de 1 min em 100\% da potência pico, intercalados por $75 \mathrm{~s}$ de recuperação) por 2 semanas também elevaram o conteúdo de GLUT4 ${ }^{67}$. Adicionalmente, apenas uma semana de esforços de $30 \mathrm{~s}$ all-out em bicicleta ergométrica, alternados por 4 min de recuperação, já aumentou a quantidade de GLUT4 muscular e, após uma semana de destreinamento, esta quantidade se manteve $20 \%$ superior à situação de baseline $^{68}$. Estes achados evidenciam que o treinamento intenso de baixo volume pode rapidamente melhorar o controle glicêmico e induzir adaptações musculares associadas à saúde metabólica em pacientes com DM2.

Complementarmente, pacientes com obesidade, HAS, DCV, DM2 e RI apresentam níveis baixos de adiponectina e o aumento da concentração desta proteína no plasma está associado à melhora da tolerância a glicose e da SI. Neste contexto, Moghadasi et al. (2011) ${ }^{63}$ evidenciaram que 12 semanas de treinamento intenso (80\% $\mathrm{VO}_{2}$ max por $45 \mathrm{~min}$ ) elevaram adiponectina e reduziram a $\mathrm{RI}$ em adultos obesos.

Reforçando o conceito de eficiência temporal, Metcalfe et al. $(2011)^{64}$ avaliaram as respostas orgânicas decorrentes de uma versão reduzida de HIIT (RE-HIIT) para identificar a dose mínima necessária para obtenção de benefícios à saúde. Nesta investigação, 3 sessões/sem de apenas 10 min foram executadas durante seis semanas ( 2 x 10-20s all-out, com intervalos de aproximadamente $3 \mathrm{~min})$ e foram suficientes para promover aumento da SI (28\%) e do $\mathrm{VO}_{2}$ pico (15\%). Estes resultados indicaram que o RE-HIIT parece ser capaz de melhorar a saúde metabólica e a capacidade aeróbia de maneira temporalmente eficiente e se constitui como uma alternativa aos protocolos convencionais para tratamento de DM2. Neste sentido, estudo recente registrou que 4 sessões semanais de HIIT, com esforços all-out realizados de modo intermitente ( 8 x $20 \mathrm{~s}$ x $10 \mathrm{~s}$ de recuperação passiva, total de $16 \mathrm{~min} / \mathrm{sem})$, geraram ganhos de $7 \mathrm{a} 8 \%$ no $\mathrm{VO}_{2} \max$, semelhantes à realização de 30 min de exercícios contínuos em 85\% da FCmax, com total de 120 min semanais ${ }^{65}$. 


\section{Obesidade}

De maneira crônica, o HIIT influencia o balanço lipídico favorecendo a redução da gordura corporal ${ }^{48}$. Confirmando esta afirmação, houve maior redução da gordura subcutânea decorrente do HIIT (esforços curtos = 10 a 15 tiros de 10 a $15 \mathrm{~s}$ a $60 \%$ do trabalho máximo mensurado em teste de $10 \mathrm{~s}$ e estímulos longos $=5 \mathrm{a}$ 6 tiros de 60 a 90 s a $70 \%$ do trabalho máximo mensurado em teste de 90 s; com recuperação até a FC atingir de 120 a $130 \mathrm{bpm}$ ) do que de um protocolo contínuo (30 a 45 min entre $60 \%$ e $85 \%$ da FC de reserva) ${ }^{53}$. Resultado semelhante foi encontrado por Trapp et al. $(2008)^{50}$ com mulheres jovens inativas e saudáveis após 15 semanas de treinamento. Quando comparado ao grupo que executou exercício contínuo e moderado (60\% $\mathrm{VO}_{2}$ pico durante $20-40 \mathrm{~min}$ ), o grupo que fez HIIT ( 60 x 8 s all-out $\times 12$ s 20-30 rpm) apresentou reduções maiores na gordura corporal total, gordura subcutânea da coxa e gordura abdominal, que podem estar associadas à supressão de apetite e/ou aumento da utilização lipídica.

Adicionalmente, resultados encontrados por Irving et al. (2008) ${ }^{49}$ indicam que o exercício intenso é mais eficiente que o moderado para alterar a composição corporal de mulheres obesas portadoras de SM, pois foi evidenciada maior redução da gordura subcutânea abdominal $\left(-47 \mathrm{~cm}^{2}\right.$ vs $\left.-11 \mathrm{~cm}^{2}\right)$ e da gordura visceral abdominal $\left(-24 \mathrm{~cm}^{2} v s-7 \mathrm{~cm}^{2}\right)$ com este treinamento. Por outro lado, Gutin et al. $(2002)^{54}$ afirmam não haver efeito claro da intensidade do exercício na melhora da composição corporal e adiposidade visceral de jovens (13 a 16 anos) obesos que executaram exercícios contínuos em diferentes intensidades (75 a 80\% $\mathrm{VO}_{2}$ pico e 50 a $60 \% \mathrm{VO}_{2}$ pico), o que aparentemente denota diferença nas respostas à intensidade do exercício relacionada à idade.

Além disso, após dois meses de HIIT, alternando 60 e $90 \%$ do $\mathrm{VO}_{2}$ max a cada 4 min durante $32 \mathrm{~min}$, foram evidenciadas reduções de $28 \%$ nas taxas de VLDL e TG, tanto nas concentrações de jejum, quanto na taxa de secreção hepática. A relevância destes achados está ligada pela influência na redução dos riscos relacionados às altas concentrações de gordura no fígado (esteatose hepática) e hipertrigliceridemias ${ }^{52}$.

Em outra perspectiva, a adiposidade abdominal está associada à redução na secreção do hormônio do crescimento (GH). Neste sentido, Irving et al. (2009) $)^{51}$ relataram que 16 semanas de treinamento de alta $(3 \mathrm{~d} /$ sem acima do LL e $2 \mathrm{~d} / \mathrm{sem}$ abaixo do LL) e moderada intensidades ( $5 \mathrm{~d} / \mathrm{sem}$ abaixo do LL), aumentaram a secreção noturna de $\mathrm{GH}$ (65 vs 49\%, respectivamente) e promoveram alterações favoráveis na composição corporal, independentemente da intensidade. Estes dados suportam a ideia da aplicação de exercícios para potencializar a secreção do referido hormônio e reduzir riscos a saúde em adultos obesos portadores de SM. Resultado semelhante foi evidenciado com o exercício contínuo (30min entre LL e $\mathrm{VO}_{2}$ pico) e intervalado ( $3 \times 10$ min entre $\mathrm{LL} \mathrm{e} \mathrm{VO}_{2}$ pico x 10min), sugerindo que tanto exercícios contínuos quanto intermitentes são efetivos no aumento da secreção de GH 24 h pós-treino ${ }^{69}$.

Complementarmente, Boutcher $(2011)^{33}$ relatou que os mecanismos para perda de gordura induzida pelo HIIT incluem aumento da oxidação de gordura durante e, principalmente, pós-exercício, redução da sensação de fome, aumento da capacidade de oxidação de AGL no músculo esquelético pela demanda de remoção de lactato e íons de hidrogênio $(\mathrm{H}+)$ e de ressíntese de glicogênio, níveis elevados de GH e melhora na SI.

Cabe ressaltar, entretanto, que dos estudos analisados, apenas dois apresentaram equivalência de demanda metabólica (calorias/sessão) entre os protocolos intermitente e contínuo utilizados na comparação e, como este controle relevante 
para a interpretação das respostas, sugere-se que estudos futuros que comparem diferentes modos de exercício e/ou diferentes intensidades considerem esta variável.

\section{Indicações do HIIT para os componentes da SM}

Nesta parte do texto será considerada a aplicabilidade prática do HIIT em cada um dos componentes da SM, a qual é sintetizada no quadro 3.

Quadro 3 - Síntese dos efeitos do HIIT nas variáveis da Síndrome Metabólica.

\begin{tabular}{|c|c|}
\hline $\begin{array}{l}\text { Variável de interesse e } \\
\text { respectiva referência }\end{array}$ & Principais desfechos observados \\
\hline \multicolumn{2}{|l|}{ Composição Corporal } \\
\hline Irving et al. (2009) ${ }^{51}$ & $\downarrow$ Circunferência da cintura; $\downarrow M C ; \downarrow I M C ; \downarrow M G ; \downarrow G A ; \downarrow G V A$ \\
\hline Gutin et al. (2002) $)^{54}$ & $\downarrow G V A ; \downarrow$ Adiposidade total \\
\hline Irving et al. (2008) ${ }^{49}$ & 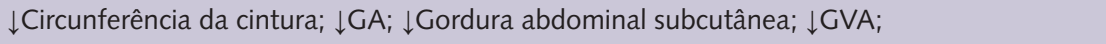 \\
\hline Moghadasi et al. $(2011)^{63}$ & $\downarrow G A ; \downarrow G V A ; \downarrow$ Gordura subcutânea; $\downarrow M C$ \\
\hline \multicolumn{2}{|l|}{ Cardiovascular } \\
\hline Tsekouras et al. $(2008)^{52}$ & $\uparrow \mathrm{VO}_{2}$ pico $(18 \%)$ \\
\hline Metcalfe etl al. $(2011)^{64}$ & $\mathrm{H}=\uparrow \vee \mathrm{O}_{2}$ pico $(15 \%) ; M=\uparrow \mathrm{VO}_{2}$ pico $(12 \%)$ \\
\hline Babraj et al. $(2009)^{30}$ & $\uparrow \mathrm{VO}_{2}$ pico $(6 \%)$ \\
\hline Gutin et al. $(2002)^{54}$ & $\uparrow \mathrm{VO}_{2}$ máx-170; $\uparrow \mathrm{VO}_{2}$ máx \\
\hline Freyssin et al. $(2012)^{56}$ & $\uparrow \mathrm{VO}_{2}$ pico $(27 \%) ; \uparrow \mathrm{VO}_{2}-\mathrm{LV} 1$ (22\%); $\uparrow$ Captação de $\mathrm{O}_{2}$ \\
\hline Astorino et al. $(2011)^{57}$ & $\uparrow \mathrm{VO}_{2}$ máx; $\uparrow \mathrm{VCO}_{2}$ máx; $\uparrow$ Captação de $\mathrm{O}_{2}$ \\
\hline Irving et al. (2009) 51 & $\uparrow \mathrm{VO}_{2}$ pico; \\
\hline Irving et al. $(2008)^{49}$ & $\uparrow \mathrm{VO}_{2}$ pico; \\
\hline Moghadasi et al. $(2011)^{63}$ & $\uparrow \mathrm{VO}_{2}$ máx; \\
\hline Trilk et al. $(2011)^{55}$ & 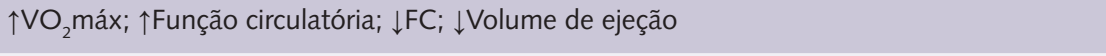 \\
\hline Tabata et al. (1996) ${ }^{80}$ & $\uparrow \mathrm{VO}_{2}$ máx; $\uparrow$ Capacidade anaeróbia \\
\hline \multicolumn{2}{|l|}{ Metabólico } \\
\hline Tsekouras et al. $(2008)^{52}$ & $\downarrow$ VLDL-TG (28\%) \\
\hline Metcalfe etl al. $(2011)^{64}$ & $\mathrm{H}=\uparrow S I(28 \%)$ \\
\hline Babraj et al. (2009) ${ }^{30}$ & $\downarrow$ AGL $(17 \%) ; \uparrow S I(23 \%) /$ Área abaixo da curva- $\downarrow$ GLI (12\%); $\downarrow$ Insulina (37\%); $\downarrow$ AGL (26\%) \\
\hline Weltman et al. $(2008)^{69}$ & $\uparrow \mathrm{GH}$ \\
\hline Irving et al. (2009) ${ }^{51}$ & $\uparrow G H(65 \%)$ \\
\hline Little et al. $(2011)^{62}$ & $\downarrow$ GLI 24h pós-treino (13\%); $\downarrow$ GLI pós-prandial (30\%); $\uparrow$ GLUT4 (369\%) \\
\hline Gillen et al. $(2012)^{43}$ & $\downarrow$ Tempo em hiperglicemia; $\downarrow$ Pico glicêmico pós-prandial; $\downarrow$ Glicemia 60 - 120 min pós-prandial \\
\hline Moghadasi et al. $(2011)^{63}$ & $\uparrow$ Expressão do RNAm de adiponectina (57\%); $\downarrow$ GLI-jejum; $\downarrow$ Insulina; $\downarrow$ RI \\
\hline Rubin et al. $(2003)^{82}$ & $\uparrow \mathrm{GH}$ \\
\hline Hood et al. $(2011)^{81}$ & 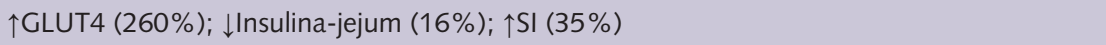 \\
\hline
\end{tabular}

$M C=$ Massa corporal; $M G=$ Massa gorda; $\mathrm{Rl}=$ Resistência insulínica; VO2máx= Consumo máximo de oxigênio; VCO2máx= Consumo máximo de gás carbônico; $\mathrm{TG}=$ Triglicerídeos; $\mathrm{VLDL}=$ lipoproteína de muito baixa densidade; $\mathrm{GH}=$ Hormônio do crescimento; $\mathrm{SI}=$ sensibilidade a insulina; $\mathrm{H}=$ Homens; $M=$ Mulheres; $\mathrm{GLI}=$ Glicose; $\mathrm{AGL}=$ Ácido Graxos Livres; VO2máx-170= Consumo máximo de oxigênio a 170 bpm; VO2-LV1= Consumo de oxigênio na intensidade do limiar ventilatório 1; IMC= Índice de massa corporal; GVA= Gordura visceral abdominal; $\mathrm{GA}=$ Gordura abdominal. 
Os efeitos do HIIT na redução da gordura subcutânea e abdominal são promissores no tratamento do sobrepeso e, consequentemente, na redução da circunferência da cintura que é um marcador de risco cardíaco ${ }^{33}$.

Em relação à HAS, as recomendações não farmacológicas para o seu tratamento envolvem dieta e exercício aeróbio de intensidade moderada em função da redução da pressão arterial sistólica e diastólica e melhora na função arterial causadas por este treinamento ${ }^{71}$. Porém, protocolos de alta intensidade com menor demanda temporal e maior grau motivacional promoveram estas adaptações em obesos sedentários ${ }^{55} \mathrm{e}$ em doentes $\operatorname{cardíacos}^{56}$. Assim, sugere-se que estudos com maiores níveis de evidência devem ser conduzidos com pacientes hipertensos.

A RI parece ser um fator fisiopatológico relevante da DM2, além de estar relacionada com outros problemas de saúde pública atual, como a obesidade e as $\mathrm{DCV}^{72}$. Recomendações para seu combate englobam exercícios de alto volume e intensidade moderada, visando atingir aumento de desempenho físico, aumento da capacidade oxidativa e melhora do mecanismo de transporte da glicose ${ }^{13}$. Porém, exercícios intermitentes de alta intensidade têm mostrado resultados satisfatórios com maior eficiência temporal ${ }^{57,72}$.

Para portadores de DM, os exercícios físicos devem promover as seguintes ações: i) auxílio na manutenção da massa corporal magra e redução da massa gorda e; ii) melhora da função cardiovascular, da SI, do perfil lipídico, do controle glicêmico e da pressão arterial ${ }^{73}$. Portanto, entende-se que a partir dos protocolos intermitentes anteriormente citados, o HIIT parecem ser mais eficientes para atender estas demandas ${ }^{50,62,66}$, contrastando com as recomendações tradicionais de esforços contínuos com duração de 20 a 60 min e intensidade entre $50 \%$ e $80 \%$ do $\mathrm{VO}_{2}$ de reserva ${ }^{73}$.

Quanto à função cardiovascular, o HIIT parece ter efeito importante ${ }^{74-75}$, principalmente por aumentar o limiar ventilatório, o que apresenta relevância clínica por melhorar a capacidade de suportar exercícios submáximos e a percepção de qualidade de vida. Além disso, o HIIT pode induzir regressão de marcadores de disfunção no ventrículo esquerdo, diminuir a resistência vascular e a disfunção endotelial e aumentar a capacidade oxidativa dos músculos periféricos ${ }^{56}$.

\section{CONCLUSÕES}

Os exercícios intermitentes de alta intensidade se mostram úteis no tratamento de fatores que caracterizam a SM. Além da eficiência temporal, da motivação e maior aderência ao processo de treinamento, a otimização dos resultados em prazos menores que os promovidos em exercícios contínuos tornam o HIIT um meio de treino interessante a ser adicionado na prescrição de exercício para esta população.

Especificamente quanto ao processo de treinamento, sugere-se que sedentários portadores de síndrome metabólica sigam uma progressão linear de intensidades e regressão concomitante do tempo dos estímulos, visando a introdução gradativa de exercícios intensos na rotina de treinamento. Esta introdução deve respeitar a tolerância em diferentes estágios, a saber:

1) Protocolos intermitentes longos e submáximos, como os de Bartlett et al. $(2011)^{76}, 6$ × 3 min a $90 \% \mathrm{VO}_{2} \max$ com intervalos de 3 min a $50 \% \mathrm{VO}_{2}$ max ou de Mandroukas et al. $(2011)^{40}, 4 \mathrm{~min}$ a $12 \mathrm{~km} / \mathrm{h} \mathrm{x} 4 \mathrm{~min}$ a $8 \mathrm{~km} / \mathrm{h}$, podem servir como introdução aos novos estímulos ainda que coexistindo com os exercícios moderados, longos e contínuos. 
2) A medida que a aptidão física aumenta, esforços intermitentes mais curtos e próximos da intensidade máxima, como os de Little et al. $(2011)^{62}, 10$ x 60s em $90 \%$ FCmax x 60 s recuperação podem potencializar os resultados e apresentar redução da demanda temporal das sessões.

3) Seguindo a progressão, indivíduos portadores de SM já treinados podem alternar os protocolos anteriormente mencionados com sessões de intensidade supramáxima, como as descritas por Trapp et al. $(1999)^{50} 60 \times 8 \mathrm{~s}$ all-out $\mathrm{x} 12 \mathrm{~s}$ a 20-30 rpm, ou de Metcalfe et al. (2011) ${ }^{64} 2 \times 10-20$ s all-out por 3 min e 20 $\mathrm{s}$ de recuperação, ou ainda Richards et al. $(2010)^{47}, 4$ x $30 \mathrm{~s}$ all-out por $4 \mathrm{~min}$ de recuperação.

Faz-se necessário reforçar que esta indicação segue uma lógica linear de progressão do treinamento e que métodos não lineares também podem ser considerados para prescrição do HIIT.

Em resumo, com o aumento da prevalência da SM, exercícios intermitentes de alta intensidade parecem ser uma alternativa relevante para a prevenção e o tratamento dos fatores de risco que a compõem. Desta forma, diferentes protocolos de treinamento de alta intensidade com potencial motivacional e eficiência temporal podem aumentar a adesão aos programas de exercícios que objetivam reduzir os fatores de risco das doenças crônicas, estando eles isolados ou agrupados (SM). Sugere-se, portanto que tal modo de exercício seja considerado na prescrição e nas recomendações de atividades para promoção da saúde na SM.

\section{Contribuições dos autores}

Fabricio B Del Vecchio contribuiu na concepção do artigo, em sua organização e delineamento estrutural, na recuperação das informações e com a revisão geral. Leony M Galliano e Victor S Coswig contribuíram na concepção e redação do artigo, na inserção dos diferentes resultados dos estudos previamente publicados e com a revisão geral do texto. Os autores declaram não haver conflitos de interesse.

\section{REFERÊNCIAS}

1. Grundy S, Brewer H, Cleeman J, Smith S, Lenfant C. Definition of metabolic syndrome. NHLBI/AHA conference proceedings. Circulation. 2004;109:433-438.

2. Balkau B, Charles M, Drivsholm T, et al. Frequency of the WHO metabolic syndrome in European cohorts, and an alternative definition of an insulin resistance syndrome. Diabetes Metab. 2002;28:364-376.

3. I Diretriz Brasileira de Diagnóstico e Tratamento da Síndrome Metabólica. Arq Bras Cardiol. 2005;Supl 1:84.

4. Ford E, Giles W, Mokdad A. Increasing prevalence of the metabolic syndrome among U.S. adults. Diab Care. 2004;27:2444-2449.

5. Laursen P,Jenkins D. The scientific basis for high-intensity interval training optimising training programmes and maximising performance in highly trained endurance athletes. Sports Med 2002;32:53-73.

6. Seligman B, Polanczyk C, Santos A, et al. Intensive practical lifestyle intervention improves endothelial function in metabolic syndrome independent of weight loss: a randomized controlled trial. Metabolis. 2011;60:1736-1740.

7. Balducci S, Zanuso S, Nicolucci A, et al. Effect of an intensive exercise intervention strategy on modifiable cardiovascular risk factors in subjects with type 2 diabetes mellitus: a randomized controlled trial: the Italian Diabetes and Exercise Study (IDES). Arch Intern Med. 2010;8:1794-1803.

8. Thomas T, Warner S, Dellsperger K, et al. Exercise and the metabolic syndrome with weight regain. J Appl Physiol. 2010;109:3-10. 
9. Bateman L, Slentz C, Willis L, et al. Comparison of aerobic versus resistence exercise training effects on metabolic syndrome (from the Studies of a Target Risk Reduction Intervention Though Defined Exercise - STRRIDE - AT/RT). Am J Cardiol. 2011;108:838-844.

10. Jurca R, Lamonte M, Church T, et al. Associations of muscle strength and aerobic fitness with metabolic syndrome in men. Med \& Sci Sports \& Exerc. 2004;36:1301-1307.

11. Mecca M, Moreto F, Burini F, et al. Ten-week lifestyle changing program reduces several indicators for metabolic syndrome in overweight adults. Diabetol Metab Syndr. 2012;19:1.

12. Rennie K, McCarthy N, Yazdgerdi S, Marmot M, Brunner E. Association of metabolic syndrome with both vigorous and moderate physical activity. Int J Epidemiol. 2003;32:600-606.

13. Schneider S, Morgado A. Effects of fitness and physical training on carbohydrate metabolism and associated cardiovascular risk factors in patients with diabetes. Diab Reviews. 1995;3:378-407.

14. American College of Sports Medicine. ACSM stand position on the appropriate intervention strategies for weight loss and prevention of weight regain for adults. Med Sci Sports Exerc. 2001;33:2145-2156.

15. Prado E, Dantas E. Efeitos dos exercícios físicos aeróbio e de força nas lipoproteínas HDL, LDL e Lipoproteína (a). Arq Bras Cardiol. 2002;79:429-433.

16. Durstine J, Haskell W. Effects of exercise on plasma lipids and lipoproteins. Exerc Sport Sci Rev. 1994;22:477-521.

17. Ciolac E, Morgado C, Bortoloto L, et al. Exercício intervalado é melhor que exercício contínuo para diminuir pressão arterial 24 horas pós-exercício em hipertensos. Rev Soc Cardiol Est São Paulo. 2003;13:48.

18. Guimarães G, Bortolotto L, Doria E, et al. Interval exercise decrease 24h blood pressure more than continuous exercise in hypertension patients. In: Final program and abstract book XVth Scientific Meeting of the Inter-American Society of Hypertension. 2003;63.

19. Torres-Leal F, Capitani M,Tirapegui J. The effect of physical exercise and caloric restriction on the components of metabolic syndrome. BJPS. 2009;45:379-399.

20. Camhi S, Stefanick M, Katzmarzyk P, Young D. Metabolic syndrome and changes in body fat from a low-fat diet and/or exercise randomized controlled trial. Obesity (Silver Spring). 2010;18:548-554.

21. Bo S, Ciccone G, Guidi S, et al. Diet or exercise: what is more effective in preventing or reducing metabolic alterations? Eur J Endocrinol. 2008;159:685-691.

22. Talanian J, Holloway G, Snook L, et al. Exercise training increases sarcolemmal and mitochondrial fatty acid transport protein in human skeletal muscle. Am J Physiol Endocrinol Metab. 2012:180-188.

23. Tjønna A, Lee S, Rognmo Ø, et al. Aerobic interval training versus continuous moderate exercise as a treatment for the metabolic syndrome: a pilot study. Circulation. 2008;118:346-354.

24. Souza L, Virtuoso J. A efetividade de programas de exercício físico no controle do peso corporal. Rev Saúde Com. 2005;1:71-78.

25. Kubukeli Z, Noakes T, Dennis S. Training techniques to improve endurance exercise performances. Sports med. 2002;8:489-509.

26. Garber CE, Blissmer B, Deschenes MR, et al. American College of Sports Medicine position stand. Quantity and quality of exercise for developing and maintaining cardiorespiratory, musculoskeletal, and neuromotor fitness in apparently healthy adults: guidance for prescribing exercise. Med Sci Sports Exerc. 2011;43:1334-1359.

27. Mitsuhashi T, Yamada C, Iida A. Long-term detraining increases the risk of metabolic syndrome in japonese men. Tokai J Exp Chlin Med 2011;4:95-99.

28. Andrews RC, Cooper AR, Montgomery AA, et al. Diet or diet plus physical activity versus usual care in patients with newly diagnosed type 2 diabetes: the Early ACTID randomised controlled trial. Lancet. 2011;9:129-139.

29. Hawley JA, Gibala MJ. What's new since Hippocrates? Preventing type 2 diabetes by physical exercise and diet. Diabetologia. 2012;55:535-539.

30. Babraj J, Vollaard N, Keast C. Extremely short duration high intensity interval training substantially improves insulin action in young healthy males. BMC Endocr Disor 2009;3.

31. Gibala M, McGee S. Metabolic adaptations to short-term high-intensity interval training: a little pain for a lot of gain? Sports Med 2008;2:58-63. 
32. Buchheit M, Abbiss C, Peiffer J, Laursen P. Performance and physiological responses during a sprint interval training session: relationships with muscle oxygenation and pulmonary oxygen uptake kinetics. J Appl Physiol. 2012;112:767-779.

33. Boutcher SH. High-Intensity Intermittent Exercise and Fat Loss. Journal of Obesity

34. 2011;2011:1-10.

35. Peterson L, Herrero P, Schechtman K, et al. Effect of obesity and insulin resistance on myocardial substrate metabolism and efficiency in young women. Circulation. 2004;109:21912196.

36. Ha TH, Seo HS, Choo WJ, et al. The effect of metabolic syndrome on myocardial contractile reserve during exercise in non-Diabetic Hypertensive Subjects. J Cardiovasc Ultrasound. 2011;19:176-182.

37. Spencer M, Bishop D, Dawson B, Goodman C. Physiological and metabolic responses of repeated-sprint activities. Sports Med. 2005;12:1025-1044.

38. Trump M, Heigenhauser G, Putnam C, Spriet L. Importance of muscle phosphocreatine during intermittent maximal cycling. J Appl Physiol. 1996;5:1574-1580.

39. Christmass M, Dawson B, Passeretto P, Arthur P. A comparison of skeletal muscle oxygenation and fuel use in sustained continuous and intermittent exercise. J Appl Physiol. 1999;8:423-435.

40. Christmass M, Dawson B, Arthur P. Effect of work and recovery duration on skeletal muscle oxygenation and fuel use during sustained intermittent exercise. J Appl Physiol. 1999;8:436-447.

41. Mandroukas A, Heller J, Metaxas T. Cardiorespiratory and metabolic alterations during exercise and passive recovery after three modes of exercise. J Strength Cond Res. 2011;6:1664-1672.

42. Billat L. Interval training for performance: A scientific and empirical practice special recommendations for middle- and long-distance running. Part I: Aerobic interval training. Sports Med. 2001;31:13-31.

43. Currie K, Mckelvie R, Macdonald M. Flow-Mediated Dilation Is Acutely Improved after High-Intensity Interval Exercise. Med Sci Sports Exerc. 2012;44:2057-2064.

44. Gillen J, Little J, Punthakee Z. Acute high-intensity interval exercise reduces the postprandial glucose response and prevalence of hyperglycemia in patients with type 2 diabetes. Diab Obes Metab. 2012;6:575-577.

45. Einhorn D, Reaven G, Cobin R, et al. American College of Endocrinology position statement on the insulin resistance syndrome. Endocr Pract. 2003;9:237-252.

46. Bollinger L, Lafontaine T. Exercise programming for insulin resistance. Strength Cond J. 2011;5:44-47.

47. Whyte L, Ferguson C, Wilson J, Scott R, Gill J. Effects of single bout of very high-intensity exercise on metabolic health biomarkers in overweight/obese sedentary men. Metabolism. 2012; http://dx.doi.org/10.1016/j.metabol.2012.07.019:1-8.

48. Richards J, Johnson T, Kuzma J. Short-term sprint interval training increases insulin sensitivity in healthy adults but does not affect the thermogenic response to $\nabla$-adrenergic stimulation. J Physiol. 2010;15:2961-2972.

49. Yoshioka M, Doucet E, Pierre S. Impact of high-intensity exercise on energy expenditure, lipid oxidation and body fatness. Int J Obes Relat Metab Disord 2001;3:332-339.

50. Irving B, Davis C, Brock D. Effect of exercise training intensity on abdominal visceral fat and body composition. Med Sci Sports Exerc. 2008;11:1863-1872.

51. Trapp E, Chisholm D, Freund J, Boutcher S. The effects of high-intensity intermittent exercise training on fat loss and fasting insulin levels of young women. Int J Obes Relat Metab Disord. 2008;32:684-691.

52. Irving B, Weltman J, Patrie J. Effects of exercise training intensity on nocturnal growth hormone secretion in obese adults with the metabolic syndrome. J Clin Endocrinol Metab. 2009;6:1979-1986.

53. Tsekouras Y, Magkos F, Kellas Y. High-intensity interval aerobic training reduces hepatic very low-density lipoprotein-triglyceride secretion rate in men. J Physiol Endocr Metab. 2008;5:851-858.

54. Tremblay A, Simoneau JA, Bouchard C. Impact of exercise intensity on body fatness and skeletal muscle metabolism. Metabolism. 1994;43:814-818.

55. Gutin B, Barbeau P, Owens S. Effects of exercise intensity on cardiovascular fitness, total body composition, and visceral adiposity of obese adolescents. J Clin Nutrition. 2002;7:818-826. 
56. Trilk JL, Singhal A, Bigelman KA, Cureton KJ. Effect of sprint interval training on circulatory function during exercise in sedentary, overweight/obese women. Eur J Appl Physiol. 2011;111:1591-1597.

57. Freyssin C, Verkindt C, Prieur F. Cardiac rehabilitation in chronic heart failure: effect of a 8-week high-intensity interval training vs continuous training. Arch Phys Med Rehabil. 2012;8:1359-1364.

58. Astorino T, Allen R, Roberson D, Jurancich M. Effect of high-intensity interval training on cardiovascular function, VO2max, and muscular force. J Strength Cond Res. 2012;1:138-145.

59. Morikawa M, Okazaki K, Masuki S, et al. Physical fi tness and indices of lifestyle-related diseases before and after interval walking training in middle-aged and older males and females. Br J Sports Med. 2011;45:216-224.

60. Ogawa T, Spina R, Martin W. Effects of aging, sex, and physical training on cardiovascular responses to exercise. Circulation. 1992;86 494-503.

61. Lepretre $P$, Vogel T, Brechat $P$, et al. Impact of short-term aerobic interval training on maximal exercise in sedentary aged subjects. Int J Clin Pract Suppl. 2009;63:1472-1478.

62. Karstoft K,Winding K, Knudsen S, et al. The Effects of Free-Living Interval-Walking Training on Glycemic Control, Body Composition, and Physical Fitness in Type 2 Diabetes Patients. Diab Care. 2012;21:1-9.

63. Little JP, Gillen JB, Percival ME, et al. Low-volume high-intensity interval training reduces hyperglycemia and increases muscle mitochondrial capacity in patients with type 2 diabetes. J Appl Physiol. 2011;111:1554-1560.

64. Moghadasi M, Mohebbi H, Rahmani-Nia F. High-intensity endurance training improves adiponectin mRNA and plasma concentrations. Eur J Appl Physiol. 2012;4:1207-1214.

65. Metcalfe R, Babraj J, Fawkner S, Vollaard N. Towards the minimal amount of exercise for improving metabolic health: beneficial effects of reduced-exertion high-intensity interval training. Eur J Appl Physiol. 2011;7:2767-2775.

66. McRae G, Payne A, Zelt J. Extremely low volume, whole-body aerobic-resistance training improves aerobic fitness and muscular endurance in females. Appl Physiol Nutr Metab. 2012;37:1124-1131.

67. Earnest C. Exercise interval training: An improved stimulus for improving the physiology of pre-diabetes. Med Hypotheses 2008;71:752-761.

68. Little J, Safdar A, Wilkin G, Tarnopolsky M, Gibala M. A practical model of low-volume high-intensity interval training induces mitochondrial biogenesis in human skeletal muscle: potential mechanisms. J Physiol. 2010;58:1011-1022.

69. Burgomaster K, Cermak N, Phillips S, et al. Divergent response of metabolite transport proteins in human skeletal muscle after sprint intervaltraining and detraining. Am J Physiol Regul Integr Comp Physiol. 2007;292:1970-1976.

70. Weltman A, Weltman J,Winfield D. Effects of continuous versus intermittent exercise, obesity, and gender on growth hormone secretion. J Endocr Metab. 2008;12:4711-4720.

71. Jeon K, Lee O, Kim H, Han S. Comparison of the dietary intake and clinical characteristics of obese and normal weight adults. Nutr Res Pract 2011;4:329-336.

72. Collier SR, Frechette V, Sandberg K, et al. Sex differences in resting hemodynamics and arterial stiffness following 4 weeks of resistance versus aerobic exercise training in individuals with pre-hypertension to stage 1 hypertension. Biol Sex Differ. 2011;2:9.

73. Gibala M, Little J. Just HIT it! A time-efficient exercise strategy to improve muscle insulin sensitivity. J Physiol. 2010;18:3341-3342.

74. Castro A, Mansur H, Junior J. Atuação do educador físico em um programa de educação em diabetes. In: UFJF, editor. Diabetes mellitus: abordagem interdisciplinar A educação em saúde como tratamento. Juiz de Fora: UFJF; 2011. p. 98-110.

75. Kessler H, Sisson S, Short K. The Potential for High-Intensity Interval Training to Reduce Cardiometabolic Disease Risk. Sports Med. 2012;42:489-509.

76. Guiraud T, Nigam A, Gremeaux V, et al. High-intensity interval training in cardiac rehabilitation. Sports Med. 2012;42:587-605.

77. Bartlett JD, Close GL, MacLaren DPM, et al. High-intensity interval running is perceived to be more enjoyable than moderate-intensity continuous exercise: Implications for exercise adherence. Journal of Sports Sciences. 2011;29:547-553. 
78. Gosselin L, Kozlowski K, Devinneyboymel L, Hambridge C. Metabolic response of different high intensity aerobic interval exercise protocols. J Strength Cond Res. 2012;26:2866-2871.

79. Goto K, Tanaka K, Ishii N, Uchida S, Takamatsu K. A single versus multiple bouts of moderateintensity exercise for fat metabolism. Clin Physiol Funct Imaging. 2011;31:215-220.

80. Goto K, Ishii N, Mizuno A, Takamatsu K. Enhancement of fat metabolism by repeated bouts of moderate endurance exercise. J Appl Physiol. 2007;102:2158-2164.

81. Tabata I, Nishimura K, Kouzaki K. Effects of moderate-intensity endurance and highintensity intermittent training on anaerobic capacity and VO2max. Med Sci Sports Exerc. 1996;10:1327-1330.

82. Hood M, Little J, Tarnopolsky M, Myslik F, Gibala M. Low-Volume interval training improves muscle oxidative capacity in sedentary adults. Med Sci Sports Exerc. 2011;10:1849-1856.

83. Rubin M, Kraemer W, Kraemer R. Responses of growth hormone aggregates to different intermittent exercise intensities. Eur J Appl Physiol. 2003;8:166-170.

$$
\begin{array}{r}
\text { Endereço para Correspondência } \\
\text { Fabrício Boscolo Del Vecchio } \\
\text { Rua Luiz de Camões, } 625 \\
\text { Bairro Tablada } \\
\text { CEP: } 96055-630 \text { - Pelotas/RS } \\
\text { fabricio_boscolo@uol.com.br } \\
\text { (53) } 32732752
\end{array}
$$

$$
\begin{array}{ll}
\text { Recebido } & 21 / 11 / 2012 \\
\text { Revisado } & 14 / 04 / 2013 \\
& 12 / 06 / 2013 \\
\text { Aprovado } & 21 / 06 / 2013
\end{array}
$$

